\title{
Single-Molecule Total Internal Reflection Fluorescence Microscopy
}

\author{
Emily M. Kudalkar ${ }^{1}$, Trisha N. Davis ${ }^{1,}$, , and Charles L. Asbury ${ }^{2}$ \\ ${ }^{1}$ Department of Biochemistry, University of Washington, Seattle, WA 98195 \\ ${ }^{2}$ Department of Physiology and Biophysics, University of Washington, Seattle, WA 98195
}

\begin{abstract}
The advent of total internal reflection fluorescence (TIRF) microscopy has permitted visualization of biological events on an unprecedented scale: the single molecule level. Using TIRF, it is now possible to view complex biological interactions such as cargo transport by a single molecular motor or DNA replication in real-time. TIRF allows for visualization of single molecules by eliminating out-of-focus fluorescence and enhancing the signal-to-noise ratio. TIRF has been instrumental for studying in vitro interactions and has also been successfully implemented in livecell imaging. Visualization of cytoskeletal structures and dynamics at the plasma membrane, such as endocytosis, exocytosis, and adhesion, has become much clearer using TIRF microscopy. Thanks to recent advances in optics and commercial availability, TIRF microscopy is becoming an increasingly popular and user-friendly technique. In this introduction, we describe the fundamental properties of TIRF microscopy and the advantages of using TIRF for single-molecule investigation.
\end{abstract}

\section{Single Molecule Visualization Using TIRF Microscopy}

Since the development of fluorescence microscopy, a long-standing problem has been the limitation of visualizing single fluorescent molecules. Standard microscopes have a resolution limit near $0.2 \mu \mathrm{m}$, much too large to spatially distinguish individual protein complexes. Recent techniques have been developed that greatly enhance resolution, such as deconvolution and confocal microscopy, but these are still confounded by out-of-focus light or rapid photobleaching. One major problem with standard fluorescence imaging is limiting the excitation of fluorescent molecules to a precise focal plane. Out-of-focus fluorescence increases the background noise and detracts from the intensity of true signal, making spatial resolution difficult, if not impossible. The development of TIRF microscopy has effectively eliminated out-of-focus fluorescence by restricting excitation to a very thin section near the coverslip, making it possible to achieve single molecule or particle detection (Axelrod et al. 1984). This selective excitation also reduces photobleaching of fluorophores in solution and prevents harmful light damage when imaging live cells.

*Corresponding author: Telephone (206) 221-7975; tdavis@uw.edu. 


\section{Basic Physics of TIRF Microscopy}

The basic concept of TIRF microscopy applies the fundamental properties of optical physics to generate an evanescent field to excite fluorophores instead of using direct illumination. In a typical TIRF setup, light is transmitted through two adjacent materials, one of a higher refractive index (such as a glass coverslip), followed by another with a lower refractive index (such as an aqueous solution). As light passes through the media, it is partially reflected and refracted depending on the incident angle. By using two materials with different refractive indices, a critical angle can be reached: the point where all the light is refracted parallel to the interface of the two materials. Once this critical angle is passed, the phenomenon of total internal reflection (TIR) occurs (Fig. 1). Although light no longer passes through the second medium, the reflected light creates an electromagnetic field that penetrates into the less refractive material. This electromagnetic field is evanescent and decays exponentially with the depth of penetration. TIRF microscopy exploits this evanescent field, which is typically $\sim 100 \mathrm{~nm}$ thick, to exclusively excite fluorophores at the boundary of the two media, that is, very near to the coverslip. This restriction eliminates excitation of molecules away from the coverslip, thereby significantly reducing out-of-focus background fluorescence. Until recently, reaching the proper incident angle to induce TIR was rather challenging due to the limitations of commercially available microscope objectives. However, the development of new objectives with sufficiently high numerical aperture (typically $>1.45 \AA$ ) to reach the proper incident angle has made TIRF microscopy much more accessible to researchers in recent years (Axelrod 2001). This advancement combined with the increase in commercial availability of TIRF microscopes has greatly enhanced the popularity and usage of TIRF microscopy in today's research.

\section{Applications of TIRF Microscopy}

TIRF microscopy is especially useful for studying protein-protein and protein-nucleic acid biochemical interactions. TIRF permits direct visualization of binding events and quantification of kinetic on and off rates. Standard bulk biochemical assays report on the average properties of a population and often miss individual variability and stochasticity that can only be appreciated at the single molecule level. Visualizing single complexes can reveal sample heterogeneity such as the presence of different oligomeric states of a complex. TIRF can be used for stoichiometric quantification of proteins within complexes that are tagged with different fluorescent probes or by quantifying photobleach steps using single probes (Ulbrich and Isacoff 2007). Because the concentration range used in TIRF assays is often on the $\mathrm{pM}-\mathrm{nM}$ scale, experiments can be performed using minute amounts of protein or DNA. This becomes extremely useful when visualizing native complexes purified from cells since often very little material is recovered. TIRF also makes it possible to watch assembly and disassembly of macromolecular complexes and can reveal hierarchical orders that may be obscured using other techniques (Hoskins et al. 2011). Using TIRF, it is now possible to view complex biological interactions such as cargo transport by a single molecular motor or DNA replication in real-time (Axelrod et al. 1983; Vale et al. 1996; Ha et al. 1999; Tanner et al. 2009; Yardimci et al. 2010; Hoskins et al. 2011). 
Single-molecule imaging has been vital to the characterization of molecular movements along a substrate, especially polymers such as actin, microtubules, or DNA (Funatsu et al. 1995; Vale et al. 1996; Harada et al. 1999). In addition, visualization of cytoskeletal structures and dynamics at the plasma membrane, such as endocytosis, exocytosis, and adhesion, has become much clearer using TIRF microscopy (Mashanov et al. 2003; Cai et al. 2007). Molecular diffusion and movement can be difficult to see within cells due to the complexity of structures within the cytoplasm. Recapitulating these events in vitro is a powerful method used for understanding biochemical function. Visualizing molecular events in real time provides key information about their temporal regulation, such as the stalling and re-initiation that helicases undergo while unwinding DNA (Ha et al. 2002). Singlemolecule analysis is an excellent method for elucidating the function of enzymes and characterizing the timing of individual steps in catalytic reactions. TIRF has been instrumental in understanding the precise function of molecular machines such as the handover-hand motion of myosin V along microtubules (Yildiz et al. 2003), GroEl-assisted protein folding (Yamasaki et al. 1999), the dynamics of actin polymerization (Amann and Pollard 2001), and the analysis of the dynamic assembly of the spliceosome (Hoskins et al. 2011).

Coupling the properties of TIRF microscopy with other optical and biophysical techniques has been quite successful for the development of new combinatorial technologies. TIRF has been used to develop single-pair fluorescence resonance energy transfer (spFRET), giving even higher spatial resolution to single molecular co-localization (Weiss 1999). spFRET can reveal conformational changes within single enzymes during their interactions with substrates. TIRF combined with super resolution methods, such as stochastic optical reconstruction microscopy (STORM), has been useful for refining molecular localization to within a few nanometers. Coupling TIRF with optical tweezer microscopy has also been tremendously powerful. Simultaneous use of both technologies allows for direct correlation of mechanical movement with structural changes (Ishijima et al. 1998; Lang et al. 2003). These techniques have lead to important and sometimes surprising findings, such as the demonstration that ATP hydrolysis and mechanical movement are not always simultaneous (Ishijima et al. 1998).

\section{Practical Aspects for Experiments Using TIRF Micrsoscopy}

Step-by-step procedures describing how to prepare and image samples for TIRF and analyze the resulting data are presented in Protocol: Coverslip Cleaning and Functionalization for Total Internal Reflection Fluorescence Microscopy<prot085548> (Kudalkar et al. 2015a), Protocol: Preparation of Reactions for Imaging with Total Internal Reflection Fluorescence Microscopy<prot085563> (Kudalkar et al. 2015b), and Protocol: Data Analysis for Total Internal Reflection Fluorescence Microscopy<prot085571> (Asbury 2015).

\section{Acknowledgments}

The authors would like to thank Neil Umbreit and Erik Yusko for their help with developing experimental protocols. EMK, TND, YD, and CLA are supported by the NIH (F32GM099223 to EMK, R01GM040506 to TND, R01GM079373 and S10RR026406 to CLA) and YD and CLA are also supported by the Packard Fellowship (2006-30521 to CLA). 


\section{References}

Amann KJ, Pollard TD. Direct real-time observation of actin filament branching mediated by Arp2/3 complex using total internal reflection fluorescence microscopy. Proc Natl Acad Sci U S A. 2001; 98:15009-15013. [PubMed: 11742068]

Asbury CL. Data analysis for total internal reflection fluorescence microscopy. Cold Spring Harb Protoc. 2015; doi: 10.1101/pdb.prot085571

Axelrod D. Selective imaging of surface fluorescence with very high aperture microscope objectives. J Biomed Opt. 2001; 6:6-13. [PubMed: 11178575]

Axelrod D, Burghardt TP, Thompson NL. Total internal reflection fluorescence. Annu Rev Biophys Bioeng. 1984; 13:247-268. [PubMed: 6378070]

Axelrod D, Thompson NL, Burghardt TP. Total internal inflection fluorescent microscopy. J Microsc. 1983; 129:19-28. [PubMed: 6827590]

Cai D, Verhey KJ, Meyhofer E. Tracking single Kinesin molecules in the cytoplasm of mammalian cells. Biophys J. 2007; 92:4137-4144. [PubMed: 17400704]

Funatsu T, Harada Y, Tokunaga M, Saito K, Yanagida T. Imaging of single fluorescent molecules and individual ATP turnovers by single myosin molecules in aqueous solution. Nature. 1995; 374:555559. [PubMed: 7700383]

Ha T, Rasnik I, Cheng W, Babcock HP, Gauss GH, Lohman TM, Chu S. Initiation and re-initiation of DNA unwinding by the Escherichia coli Rep helicase. Nature. 2002; 419:638-641. [PubMed: 12374984]

Ha T, Ting AY, Liang J, Caldwell WB, Deniz AA, Chemla DS, Schultz PG, Weiss S. Single-molecule fluorescence spectroscopy of enzyme conformational dynamics and cleavage mechanism. Proc Natl Acad Sci U S A. 1999; 96:893-898. [PubMed: 9927664]

Harada Y, Funatsu T, Murakami K, Nonoyama Y, Ishihama A, Yanagida T. Single-molecule imaging of RNA polymerase-DNA interactions in real time. Biophys J. 1999; 76:709-715. [PubMed: 9929475]

Hoskins AA, Friedman LJ, Gallagher SS, Crawford DJ, Anderson EG, Wombacher R, Ramirez N, Cornish VW, Gelles J, Moore MJ. Ordered and dynamic assembly of single spliceosomes. Science. 2011; 331:1289-1295. [PubMed: 21393538]

Ishijima A, Kojima H, Funatsu T, Tokunaga M, Higuchi H, Tanaka H, Yanagida T. Simultaneous observation of individual ATPase and mechanical events by a single myosin molecule during interaction with actin. Cell. 1998; 92:161-171. [PubMed: 9458041]

Kudalkar EM, Deng Y, Davis TN, Asbury CL. Coverslip cleaning and functionalization for total internal reflection fluorescence microscopy. Cold Spring Harb Protoc. 2015a; doi: 10.1101/ pdb.prot085548

Kudalkar EM, Davis TN, Asbury CL. Preparation of reactions for imaging with total internal reflection fluorescence microscopy. Cold Spring Harb Protoc. 2015b; doi: 10.1101/pdb.prot085563

Lang MJ, Fordyce PM, Block SM. Combined optical trapping and single-molecule fluorescence. J Biol. 2003; 2:6. [PubMed: 12733997]

Mashanov GI, Tacon D, Knight AE, Peckham M, Molloy JE. Visualizing single molecules inside living cells using total internal reflection fluorescence microscopy. Methods. 2003; 29:142-152. [PubMed: 12606220]

Tanner NA, Loparo JJ, Hamdan SM, Jergic S, Dixon NE, van Oijen AM. Real-time single-molecule observation of rolling-circle DNA replication. Nucleic Acids Res. 2009; 37:e27. [PubMed: 19155275]

Ulbrich MH, Isacoff EY. Subunit counting in membrane-bound proteins. Nat Methods. 2007; 4:319321. [PubMed: 17369835]

Vale RD, Funatsu T, Pierce DW, Romberg L, Harada Y, Yanagida T. Direct observation of single kinesin molecules moving along microtubules. Nature. 1996; 380:451-453. [PubMed: 8602245]

Weiss S. Fluorescence spectroscopy of single biomolecules. Science. 1999; 283:1676-1683. [PubMed: 10073925] 
Yamasaki R, Hoshino M, Wazawa T, Ishii Y, Yanagida T, Kawata Y, Higurashi T, Sakai K, Nagai J, Goto Y. Single molecular observation of the interaction of GroEL with substrate proteins. J Mol Biol. 1999; 292:965-972. [PubMed: 10512696]

Yardimci H, Loveland AB, Habuchi S, van Oijen AM, Walter JC. Uncoupling of sister replisomes during eukaryotic DNA replication. Mol Cell. 2010; 40:834-840. [PubMed: 21145490]

Yildiz A, Forkey JN, McKinney SA, Ha T, Goldman YE, Selvin PR. Myosin V walks hand-over-hand: single fluorophore imaging with 1.5-nm localization. Science. 2003; 300:2061-2065. [PubMed: 12791999] 


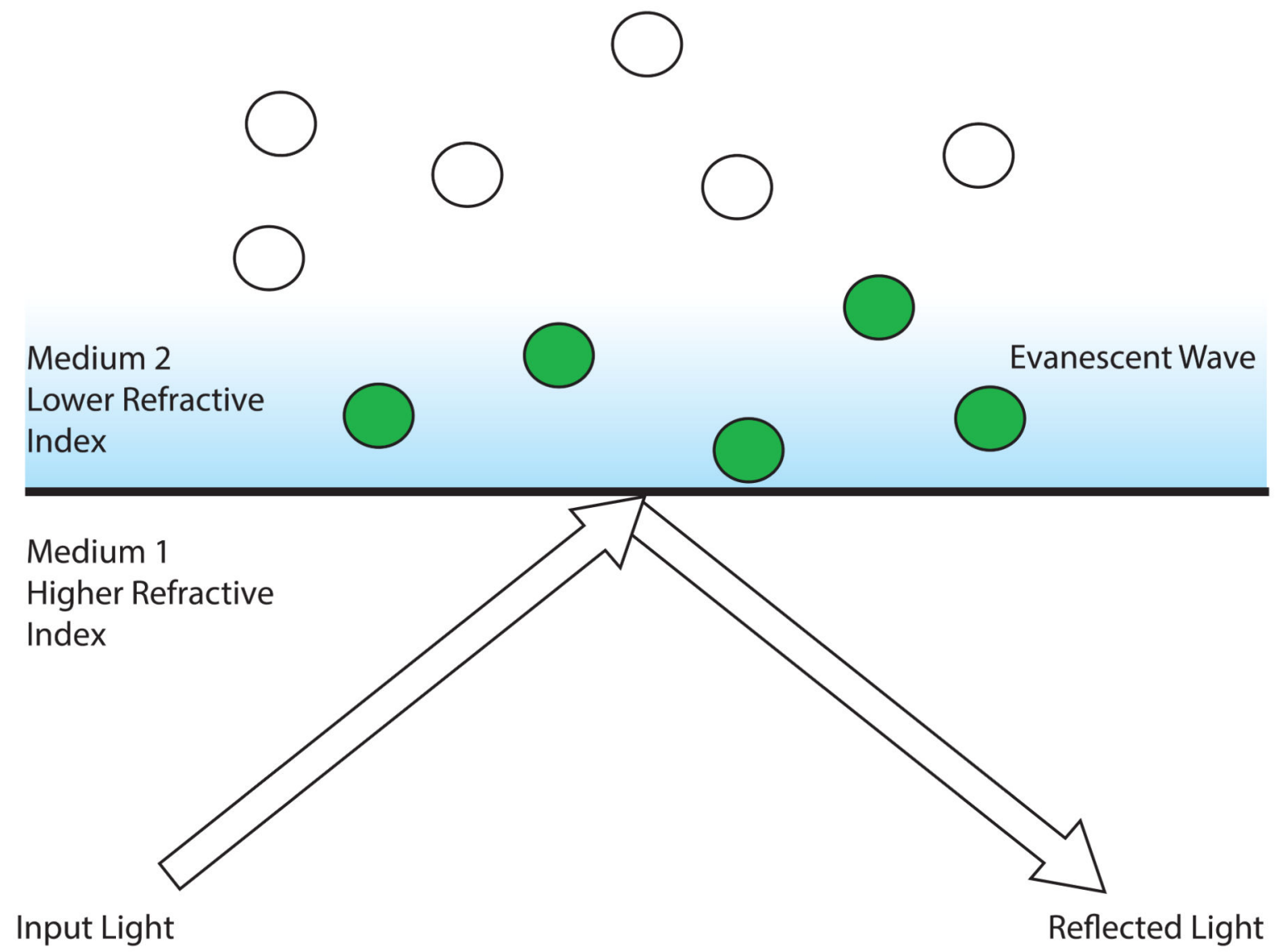

Figure 1.

Total internal reflection (TIR) microscopy. The proportion of refracted to reflected light can be changed when passing light through two different materials: one of higher refractive index and the other of lower refractive index. A critical angle can be reached that defines the point where all the light is refracted parallel to the boundary between the two mediums. Once this critical angle is passed, light is totally internally reflected and creates an evanescent wave that transmits into the second medium and decays exponentially. In total internal reflection fluorescence (TIRF) microscopy, this phenomenon is exploited to restrict illumination of fluorophores to only those within $\sim 100 \mathrm{~nm}$ of the coverslip (green spheres) while eliminating background excitation of fluorophores in solution (white spheres). 